\title{
Polarization and phase-shift properties of high spatial frequency holographic gratings in a photopolymerizable glass
}

\author{
Óscar Martínez-Matos, ${ }^{1, *}$ José A. Rodrigo, ${ }^{1}$ María L. Calvo, ${ }^{1}$ and Pavel Cheben ${ }^{2}$ \\ ${ }^{1}$ Departamento de Óptica, Facultad de Ciencias Físicas, Universidad Complutense de Madrid, 28040, \\ Madrid, Spain \\ ${ }^{2}$ Institute for Microstructural Sciences, National Research Council Canada, KlA oR6 Ottawa, Canada \\ *Corresponding author: omartine@fis.ucm.es
}

Received October 15, 2008; revised December 11, 2008; accepted January 6, 2009; posted January 15, 2009 (Doc. ID 102359); published February 11, 2009

\begin{abstract}
Polarization properties of transmission volume holographic phase gratings recorded in a photopolymerizable glass modified with high refractive index species are reported. The gratings are recorded by the interference of two parallel $s$-polarized writing beams with orthogonal propagation directions. High optical quality, low scattering, and diffraction efficiency of $99.4 \%$ are achieved. Degrees of polarization of 0.987 and 0.999 are obtained for transmitted and diffracted light, respectively. Furthermore, phase analysis of the transmitted light reveals a phase discontinuity of $\pi$ at the Bragg angle. (C) 2009 Optical Society of America

OCIS codes: 090.2890, 090.7330, 050.7330, 160.6060, 160.2900.
\end{abstract}

The polarization properties of volume holographic gratings have been studied since the pioneering work by Kogelnik in 1969 [1]. Interesting applications of the polarizing Bragg gratings, such as holographic polarizing beam splitters (HPBS) [2,3], switches and couplers [4], and diffractive optical elements incorporated in astronomical spectrographs [5], have been proposed. A good performance of these optical elements can be achieved providing holographic gratings separating the incident beam into two orthogonal polarization states, typically $s$ and $p$, with large extinction ratios. Kogelnik's theory studies the electromagnetic wave for each polarization component, $s$ and $p$, propagating independently in the medium with the respective coupling coefficients defined by [1]: $\kappa_{P}=\kappa_{S} \cos \left(2 \theta_{B}\right)$, where $\theta_{B}$ is the Bragg angle inside the medium ( $2 \theta_{B}$ is the angle between the two recording beams). Interesting grating characteristics are obtained in a $90^{\circ}$ recording geometry, say $2 \theta_{B}$ $=90^{\circ}$. For this case $\kappa_{P}=0$ and therefore the diffraction efficiency of the polarization state $p$ vanished. The incident beam is totally transmitted through the holographic sample. On the other hand, the $s$-polarized beam is diffracted with an efficiency $\eta(\theta)$, where $\theta$ is the incidence angle of the reading beam inside the medium. For the particular case $\eta\left(\theta_{B}\right)=1$, the grating splits the incident light into two beams with orthogonal polarization states, thus acting as an HPBS. However, this condition is rather restrictive, since the holographic grating has to satisfy a specific ratio between the sample thickness $T$ and the refractive index modulation $\Delta n$ [1].

Several holographic materials, such as dichromated gelatins [2,5,6], photopolymers [7], and silver halides [3], have been used to record polarizing gratings in a $90^{\circ}$ recording geometry. Typical challenges arising in some of these materials are shrinkage of the fringe pattern during the development process, low diffraction efficiency, grating defects, low values of the refractive index modulation, and optical scattering. A holographic material with a high dynamic range, low scattering, minimized shrinkage, the ability to record high spatial frequency gratings, and good mechanical as well as thermal stability is required for recording polarizing gratings with a high optical quality.

In this Letter we report the first study, to the best of our knowledge, of polarization properties of a volume transmission phase grating in a photopolymerizable glass [8]. This material [8] is a modified composition of a highly efficient photopolymerizable solgel glass synthesized earlier by Cheben et al. [9] wherein the modification is achieved by incorporating the Zr-based high refractive index species (HRIS) at the molecular level. The physical mechanism of grating formation in this glass involves a codirectional diffusion of monomer and HRIS species upon inhomogeneous illumination [10]. This mechanism results in a self-developed hologram with a permanent phase grating with an increased dynamic range along with low coherent and incoherent scattering noise, high optical quality, high diffraction efficiency, and negligible shrinkage [8]. The optical Pendellösung effect was reported in the visible domain using this material [11].

We holographically recorded transmission volume gratings in a $90^{\circ}$ recording geometry by the interference of two collimated mutually coherent $s$-polarized beams of $532 \mathrm{~nm}$ in wavelength and $50 \mathrm{~mW}$ of power. To achieve a $90^{\circ}$ recording geometry inside the holographic material (HM), Fig. 1(a), the photopolymerizable glass was sandwiched between two right-angle prisms (refractive index $n=1.519$ ) using an index matching liquid (Norland Index Matching Liquid $150, n=1.52$ ) providing an optical contact between the holographic recording material $(n=1.525)$ and the prisms. The recording and reading process of the 


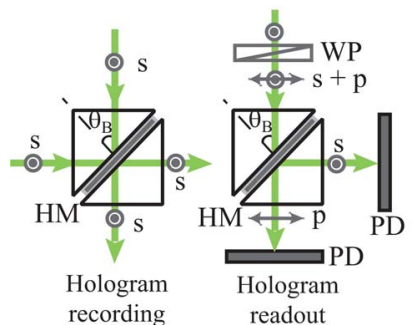

(a)

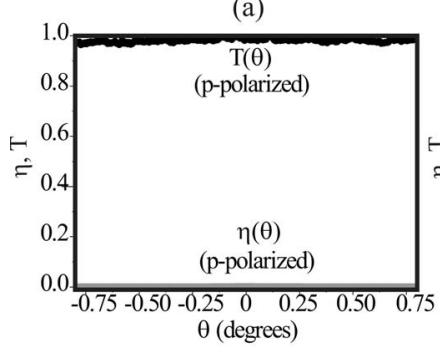

(c)

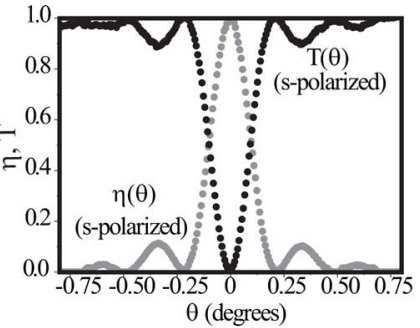

(b)

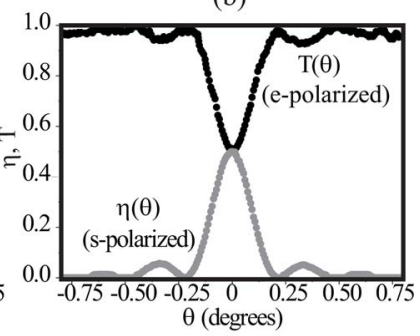

(d)
Fig. 1. (Color online) Experimental setup of holographic grating recording and readout. $\mathrm{PD}$, photodetector; WP, waveplate. Angular selectivities for transmittance $T$ (black circles) and diffraction $\eta$ (gray circles) of the $s, p$, and circularly polarized readout beams in (b)-(d), respectively.

grating is sketched in Fig. 1(a). Maximum diffraction efficiency was reached for an exposure time $t \sim 10 \mathrm{~s}$. The transmittance and the diffraction efficiency are measured for $s, p$, and circularly polarized readout beams $(\lambda=532 \mathrm{~nm})$ by introducing a half-wave plate or a quarter-wave plate in the optical path of the readout beam [Fig. 1(a)]. The sample was positioned on a precision rotation stage to measure the diffraction efficiency as a function of the incident angle $\theta$, i.e., the grating angular selectivity curves.

Figures 1(b)-1(d) show the angular selectivity curves for the diffracted $\eta(\theta)$ (gray circles) and the transmitted $T(\theta)$ (black circles) light during the hologram readout process for $s, p$, and circularly polarized readout beams, respectively. We used collimated beams in all cases. The grating spatial frequency for a $90^{\circ}$ recording geometry is 4050 lines $/ \mathrm{mm}$ and a refractive index modulation of $\Delta n=3.2 \times 10^{-3}$ was achieved for a sample with thickness $T=57 \mu \mathrm{m}$. The Klein-Cook parameter is $Q \sim 2000$. The angular selectivity curve $\eta(\theta)$ in Fig. 1(b) shows the typical diffraction behavior of a volume holographic grating, in good agreement with Kogelnik's theory. Experimental results for the transmitted beam $T(\theta)$ satisfy $T(\theta)=1-\eta(\theta)$. The symmetry of the angular selectivity curves as well as the well-defined secondary maxima and minima are remarkable. These results confirm the high quality and homogeneity of the grating. For an $s$-polarized readout beam, the maximum diffraction efficiency $99.4 \%$ was achieved under Bragg incidence. Note that this value corresponds to the relative efficiency, since there are losses by Fresnel reflection in the prisms. The insertion loss of the device is $0.7 \mathrm{~dB}$. Similar results were obtained for several gratings, ensuring reproducibility. To the best of our knowledge, this is the highest diffraction efficiency reported in related literature for holographic gratings recorded in a photopolymerizable composi- tion. The angular FWHM of the angular selectivity curve is $0.22^{\circ}$. We remark that this experimental configuration can be used as a variable beam splitter, where the splitting ratio is controlled by adjusting the angle of incidence.

The $P$-polarized readout beam is fully transmitted by the sample with diffraction suppressed, Fig. 1(c), and a constant angular selectivity curve for transmission $(T(\theta)=1)$ and diffraction $(\eta(\theta)=0)$ is obtained. For the case of circularly polarized readout, Fig. 1(d), the polarization state is represented as a superposition of $s$ and $p$ states with identical amplitudes. The $p$ component of the circularly polarized readout beam passes through the sample with diffraction suppressed while the diffracted beam is $s$ polarized independently on the angle of incidence. Measurements of the diffracted intensity for the $s$ - and $p$-polarized readout beam yields a degree of polarization of 0.999 , which corresponds to an extinction ratio $\sim 32 \mathrm{~dB}$. The grating thus operates as a holographic polarizer with a remarkably high extinction ratio. The transmitted light for a circularly polarized readout beam is in general elliptically polarized (e-polarized), thus comprising both $s$ and $p$ components. A particular case arises under the Bragg incidence in which the $s$ component of the circularly polarized readout beam is minimized in transmission. In this case, the degree of polarization of the transmitted light ( $p$ polarization) is 0.987 , thus the extinction ratio of $22 \mathrm{~dB}$. Note that for Bragg incidence the grating operates as a 50\% HPBS [see Fig. 1(d)]. Similar results are obtained for a linearly polarized reading beam with an azimuthal angle of $45^{\circ}$.

We propose an alternative method to analyze the grating properties based on the study of the phase distribution $\varphi$ of the transmitted field. As demonstrated in [12], $\varphi$ is a function of the incidence angle $\theta$. For the particular case in which the diffraction efficiency reaches the value $\eta\left(\theta_{B}\right)=1$, the phase at the Bragg angle is not defined. In fact, $\varphi\left(\theta_{B}^{ \pm}\right)= \pm \pi / 2$ near the Bragg angle $\left(\theta_{B}^{ \pm}\right)$with a corresponding phase shift $\Delta \varphi=\left|\varphi\left(\theta_{B}^{+}\right)-\varphi\left(\theta_{B}^{-}\right)\right|=\pi$. Note that this phase shift condition is rather restrictive, since the diffraction efficiency of the grating must be close to the theoretical $(\eta=1)$ limit. For $\eta<1$, the phase shift $\varphi(\theta)$ is a continuous function, approaching the Heaviside function for $\eta \rightarrow 1$. To demonstrate very high diffraction efficiency gratings as required for $\Delta \varphi=\pi$ at the Bragg angle, the grating was first illuminated with a $p$-polarized diverging Gaussian beam with a wavefront angular width from $-0.25^{\circ}$ to $0.25^{\circ}$. The transmitted intensity was registered with a CCD camera, Fig. 2(a). A p-polarized readout beam passes through the grating with diffraction suppressed; therefore, the transmitted intensity profile has a Gaussian shape as is shown in Fig. 2(d), curve I. On the other hand, an $s$-polarized diverging Gaussian beam is diffracted by the grating, yielding a modified (nonGaussian) transmitted intensity, Fig. 2(b). The central region $\left(\theta_{B}=0\right)$ in Fig. 2(b) corresponds to the wavefront part of the readout beam with incidence at 

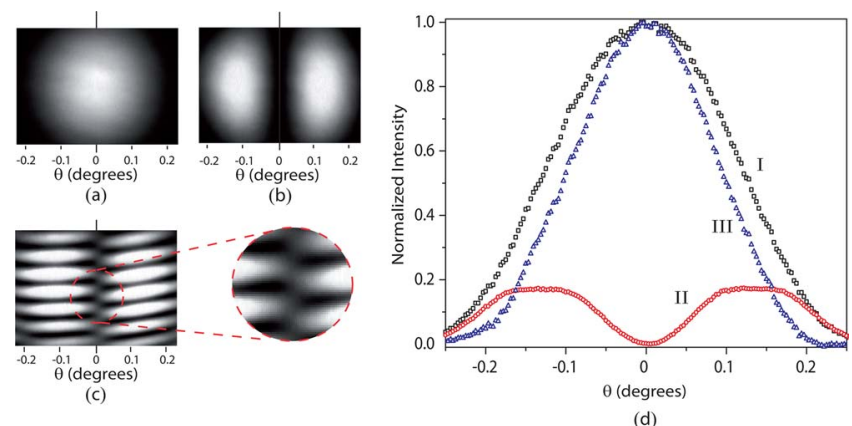

Fig. 2. (Color online) Transmitted intensity for (a) $p$-polarized and (b) $s$-polarized gratings illumination with a divergent Gaussian beam. (c) Interference pattern of (b) with an $s$-polarized collimated beam. Interferogram detail (zoom $3 \times$ ), showing the fringe interleaving. (d) Intensity profile along axis for image (a) (curve I) and image (b) (curve II). Curve III, angular dependence of diffraction efficiency of an $s$-polarized collimated beam obtained by subtraction of profiles II and I.

the Bragg angle. The light is diffracted with efficiency close to $100 \%$ for $\theta_{B}$ with a vanishing transmittance, yielding a dark region for $\theta \sim \theta_{B}$. The complementary part of the transmitted beam is symmetrically Bragg mismatched, resulting in an intensity distribution comprising two sidelobes. Its intensity profile is shown in Fig. 2(d), curve II. The phase of the transmitted $s$-polarized Gaussian field is shown by the interferogram [Fig. 2(c)] obtained by the interference with a plane wave. The interference fringe pattern in one sidelobe is a half period shifted with respect to the other sidelobe. The interleaved fringes around $\theta_{B}$ are a consequence of the $\pi$ phase shift, indicating diffraction efficiency close to $100 \%$, as discussed above. This interference method demonstrates both a very high diffraction efficiency of the holographic gratings and an excellent polarization extinction ratio, in accordance with results shown in Fig. 1. We also remark a very low scattering level of the gratings recorded in our nanocomposite glass as it is observed in the images shown in Fig. 2.

The intensity profiles in Fig. 2(d) (curves I and II) are given as a function of the incidence angle $\theta$ demonstrating a large extinction ratio for $\theta=\theta_{B}$. The angular dependence of the diffraction efficiency of an $s$-polarized readout beam, curve III in Fig. 2(d), is obtained by the subtraction of curves II and I. Curve III is in good agreement with the angular selectivity curve in Fig. 1(b). This novel method to obtain the angular selectivity simply by polarization selection of the readout beam is static, i.e., it does not involve the use of a rotation stage. The high diffraction efficiency, the polarization extinction ratio, together with the low scattering level make the gratings recorded in the photopolymerizable glass an attractive building block for fabricating polarization dependent optical elements.

We have analyzed the polarization properties of transmission volume holographic phase gratings recorded in an HRIS photopolymerizable glass for a $90^{\circ}$ recording geometry. For the first time (to our knowledge), a grating with a high spatial frequency of 4050 lines/mm was recorded in this glass type. Degrees of polarization of 0.999 and 0.987 were obtained for diffracted (s-state) and transmitted ( $p$-state) light at Bragg incidence, respectively. A diffraction efficiency of $99.4 \%$, close to the theoretical upper limit, is reached. This is among the highest diffraction efficiency values reported for holographic gratings recorded in photopolymers. We also demonstrate an alternative method for characterization of high efficiency holographic gratings based on the phase analysis of the transmitted light. It is worth highlighting the negligible scattering introduced by the grating. These results demonstrate that such gratings operate as a high performance holographic polarizer or a polarizing beam splitter, which are relevant for different applications involving polarizing and imaging elements.

We gratefully acknowledge valuable discussions with Francisco del Monte. The financial support from the Spanish Ministry of Education and Science under the project TEC 2005-02180 is also acknowledged.

\section{References}

1. H. Kogelnik, Bell Syst. Tech. J. 48, 2909 (1969).

2. S. Habraken, Y. Renotte, St. Roose, E. Stijns, and Y. Lion, Appl. Opt. 34, 3595 (1995).

3. A. M. López, M. P. Arroyo, and M. Quintanilla, J. Opt. A, Pure Appl. Opt. 1, 378 (1999).

4. R. A. Villalaz, E. N. Glytsis, and T. K. Gaylord, Appl. Opt. 41, 5223 (2002).

5. L. K. Baldry, J. Bland-Hawthorn, and J. G. Robertson, Publ. Astron. Soc. Aust. 116, 403 (2004).

6. L. D. Dickinson, R. D. Hallison, and B. H. Yung, Appl. Opt. 33, 5378 (1994).

7. J. J. Butler, M. A. Rodriguez, M. S. Malcuit, and T. W. Stone, Opt. Commun. 155, 23 (1998).

8. F. del Monte, O. Martínez-Matos, J. A. Rodrigo, M. L. Calvo, and P. Cheben, J. Adv. Mater. 18, 2014 (2006).

9. P. Cheben and M. L. Calvo, Appl. Phys. Lett. 78, 1490 (2001).

10. O. Martínez-Matos, M. L. Calvo, J. A. Rodrigo, P. Cheben, and F. del Monte, Appl. Phys. Lett. 91, 1 (2007).

11. M. L. Calvo, P. Cheben, O. Martínez-Matos, F. del Monte, and J. A. Rodrigo, Phys. Rev. Lett. 97, 084801 (2006).

12. V. Yu Bazhenov, M. V. Vasnetsov, M. S. Soskin, and V. B. Taranenko, Opt. Commun. 50, 285 (1984). 